\title{
Ill-posedness of the mean-field model of superconducting vortices and a possible regularisation
}

\author{
G. RICHARDSON ${ }^{1}$ and B. STOTH ${ }^{2}$ \\ ${ }^{1}$ School of Mathematical Sciences, University of Nottingham, Nottingham NG7 2RD, UK \\ ${ }^{2}$ Mathematisches Institut, Universität Bonn, Bonn, Germany
}

(Received 12 January 1998; revised 8 November 1999)

\begin{abstract}
We conjecture that the mean-field model of superconducting vortices given in [10] is ill-posed wherever the electric current $\mathbf{j}$ has some component in the same direction as the vorticity vector $\boldsymbol{\omega}$ (which gives the average density and direction of the superconducting vortices). The conjecture is illustrated with a linear stability analysis of a certain solution to the model. A regularised model is then proposed, and this is used to demonstrate the instability of force-free steady states in a certain geometry.
\end{abstract}

\section{Introduction}

Certain materials when cooled through a critical temperature exhibit a superconducting state in which they have the ability to conduct electric currents without resistance. A less well-known property exhibited by such materials when in this state, termed the Meissner effect, is a strong propensity to exclude magnetic fields. There is, however, a limit to a material's capacity to do this and large fields, exceeding the so-called critical field, act to destroy its superconducting properties. For fields not exceeding, but sufficiently close to, the critical field this, combined with the Meissner effect, leads to some interesting behaviour. In so-called Type-I superconductors the field penetrates the material partially through normal regions, where it is in excess of the critical field. These are then separated from the superconducting regions, in which the field is extremely small, by a sheet-like phase boundary. In Type-II superconductors, however, the field penetrates along a number of separate filaments, each associated with one quantum of magnetic flux. Around these filaments, or vortices, the superconducting properties are partially destroyed. This state, in which the magnetic field penetrates throughout the region along vortices, has been termed the mixed state, because there is no large-scale separation of normal and superconducting phases as for a Type-I superconductor.

Many models have been proposed to describe the behaviour of superconductors. One of the most successful of these is the so-called Ginzburg-Landau model [18], which describes the behaviour in terms of two variables, the magnetic field $\mathbf{H}$ and an order parameter $\psi$, the modulus of which is related to the local density of superconducting electrons. Reviews of the mathematical aspects of this model are given in $[8,15]$. Its most notable triumph was the prediction of vortices by Abrikosov [1]. An important feature 
of the model is that the morphology of solutions is found to be crucially dependent on a certain material parameter $\kappa$ which gives the ratio of typical variations in the magnetic field (the penetration depth $\lambda$ ) to typical variations in the order parameter (the coherence length $\xi)$. In particular, the value of $\kappa$ determines whether Type-I $(\kappa<1 / \sqrt{2})$ or Type-II $(\kappa>1 / \sqrt{2})$ behaviour is exhibited. Here we shall be interested in the high- $\kappa$ limit in which the thickness of the core of the vortex, outside which the size of the order parameter $\psi$ does not vary appreciably, tends to zero. For a large number of materials, typically alloys, this limit provides a good description, examples being NbSn, $\kappa=15.3 ; \mathrm{NbNi}, \kappa=28.0$ and $\mathrm{V}_{3} \mathrm{G}, \kappa=25.3$.

In applications fields are usually high enough so that vortices will appear in a Type-II superconductor, and it is therefore of importance to understand the mixed state. The dynamics of these vortices is of particular interest, since vortex motion, resulting from the flow of electric currents across vortices, leads to energy dissipation in superconductors [21]. This can cause practical problems when a transport current flows in a material and results in an effective resistivity. One of the aims to this paper is to investigate so-called force-free configurations in which a transport current flows within a material in the mixed state without causing motion of vortices [2]. To that end, we note the work of Gor'kov and Kopnin [20], which uses the Time-Dependent Ginzburg-Landau (TDGL) equations $[25,19]$, in the high- $\kappa$ limit, to derive a law of motion for a single vortex with radius of curvature much greater than the penetration depth $\lambda$. A more mathematical derivation is given in [22] and this has subsequently been generalised to cover curved vortex lines with radius of curvature an order of magnitude greater than the coherence length $\lambda / \kappa$ [11]. The result from the last of these gives the velocity $\boldsymbol{v}$ of the vortex line, in units such that the penetration depth $\lambda=1$ and the upper critical field $H_{c 2}=\kappa^{2}$, as

$$
\boldsymbol{v}=2 \mathbf{j}_{\text {ext }} \wedge \mathbf{t}+C \log (\kappa) \boldsymbol{n}+2 \mathbf{j}_{\text {fin }} \wedge \mathbf{t}+\Gamma C \boldsymbol{n}+O\left(\left|\mathbf{j}_{\text {ext }}\right| / \kappa, C \log (\kappa) / \kappa\right) .
$$

Here $\mathbf{j}_{\text {ext }}$ is that part of the current $\mathbf{j}=\nabla \wedge \mathbf{H}$ resulting from sources other than the vortex; $\mathbf{j}_{\text {fin }}$ is a non-local term arising from the vortex in question; $\Gamma$ is an $O(1)$ constant; $\mathbf{t}$ is the tangent, $C$ the curvature and $\boldsymbol{n}$ the principal normal to the vortex line. Since $\left|\mathbf{j}_{f i n}\right| \leqslant O(C)$ in cases where the vortex line does not come very close to self-intersection, it is frequently neglected, giving rise to a local velocity law of the form

$$
\boldsymbol{v}=2 \mathbf{j}_{\text {ext }} \wedge \mathbf{t}+C \log (\kappa) \boldsymbol{n} .
$$

For currents $\left|\mathbf{j}_{\text {ext }}\right| \gg C \log \kappa$ it is common practice, see for example [2], to simplify this further to

$$
\boldsymbol{v}=2 \mathbf{j}_{\text {ext }} \wedge \mathbf{t}
$$

However it has been demonstrated in [13] that vortices obeying the velocity law (1.1) can develop helical instabilities in circumstances in which $\mathbf{j}_{\text {ext }} \cdot \mathbf{t} \neq 0$. In [23] it is shown that this type of instability causes the model (1.2) to be ill-posed whenever $\mathbf{j}_{\text {ext }} \cdot \mathbf{t} \neq 0$.

Typically, the number of vortices within a sample is very large. This makes the consideration of individual vortices infeasible, and suggests that some sort of averaging technique be used to replace them with a local vortex density, or vorticity. Just such a technique has been used in [12] and [10] to arrive at a model describing the evolution of the magnetic field $\mathbf{H}$ and the vorticity $\boldsymbol{\omega}$ within a superconductor. However this model makes use of a 
vorticity velocity law based upon (1.2), and while existence and regularity of its solutions may be shown for geometries in which the current has no component in the direction of the vorticity $\nabla \wedge \mathbf{H} \cdot \boldsymbol{\omega} \equiv 0$ (see $[26,16]$ ), we conjecture that where $\nabla \wedge \mathbf{H} \cdot \boldsymbol{\omega} \neq 0$ it results in an ill-posed problem. We illustrate this conjecture in $\S 2-\S 3$ by considering the linear stability of a steady state solution, the result of which shows that the growth rate of a perturbation cannot be bounded, and thus that the problem is indeed ill-posed. The model is then modified to reinstate the curvature term in the velocity and it is found that the steady-state solution to the original model is also one for the amended model. In fact this solution describes an interesting phenomenon mentioned above, namely the "force-free steady-state" (Campbell and Evetts [2]). From a practical point of view a stable force-free steady-state is much to be desired because it allows for a non-dissipative transport current flow in the mixed state. To this end the linear stability of the steady state solution is investigated using the amended model; it is found that although the growth rate of a perturbation is bounded its size may still grow, making this solution unstable. In this context we note works by Brandt $[5,6]$ on the stability of a vortex lattice to longitudinal currents in which the onset of instability is calculated using an ad hoc model based upon the concept of 'elastic' moduli of the lattice.

\subsection{A review of the mean-field model}

In the high- $\kappa$ limit of the Ginzburg-Landau equations the thickness of a superconducting core tends to zero. Outside the core, the level of superconductivity (in terms of the Ginzburg-Landau equations, the magnitude of the order parameter $\psi$ ) is approximately constant and the Ginzburg-Landau equations simplify greatly giving rise to the so-called London equations. In a domain in which there are a number of vortex lines, lying along the curves $\Gamma_{m}$, with position vectors $\boldsymbol{x}=\boldsymbol{q}_{m}(s, t)=\left(x_{m}(s, t), y_{m}(s, t), z_{m}(s, t)\right), s$ being arclength, and where units are chosen such that the penetration depth $\lambda=1$ and the upper critical magnetic field $H_{c 2}=\kappa^{2}$, these equations may be written as follows:

$$
\begin{aligned}
\nabla^{2} \mathbf{H}_{o}-\mathbf{H}_{o} & =-\sum_{m} \boldsymbol{\delta}_{\boldsymbol{m}}(\mathbf{x}), \\
\nabla \cdot \mathbf{H}_{o} & =0
\end{aligned}
$$

where we have defined

$$
\boldsymbol{\delta}_{\boldsymbol{m}}(\mathbf{x})=2 \pi \int_{\Gamma_{m}} \delta\left(x-x_{m}(s, t)\right) \delta\left(y-y_{m}(s, t)\right) \delta\left(z-z_{m}(s, t)\right) \mathbf{d} \mathbf{d} .
$$

The motion of the vortex lines obeys the velocity law derived in [11] (see equation 1.1), the $i$ th vortex moving with velocity $\boldsymbol{v}_{i}$ given by

$$
\boldsymbol{v}_{i}=\left.2\left(\nabla \wedge \mathbf{H}_{o, i}\right)\right|_{\boldsymbol{x}=\boldsymbol{q}_{i}} \wedge \mathbf{t}_{i}+(\log \kappa) C_{i} \boldsymbol{n}_{i}+O\left(\nabla \wedge \mathbf{H}_{o, i} / \kappa, C_{i} \boldsymbol{n}_{i}\right) .
$$

Here $\mathbf{t}_{i}$ is the tangent, $C_{i}$ the curvature and $\boldsymbol{n}_{i}$ the normal to the $i$ th vortex; $\mathbf{H}_{o, i}$ is the non-singular part of the magnetic field on the line $\boldsymbol{x}=\boldsymbol{q}_{i}$ which satisfies a modified field equation, namely

$$
\nabla^{2} \mathbf{H}_{o, i}-\mathbf{H}_{o, i}=-\sum_{m \neq i} \boldsymbol{\delta}_{\boldsymbol{m}}(\mathbf{x})
$$


It is, in principle, possible to compute the evolution of the magnetic field by tracking individual vortices using equations (1.5) and (1.6). However in practice, in a sample a few millimetres across the vortices might number several million, and so any such computation on the macroscopic scale becomes prohibitively expensive. To circumvent this difficulty, Chapman [10] considers an average magnetic field and vortex density taken over a region containing many vortices. He then closes the model by finding an expression for the conservation of vorticity in terms of the velocity of the vorticity.

We now follow [10] in deriving the model. Consider a system of vortices which are separated by distances of order $\zeta$ which are small compared to the penetration depth such that $1 / \sqrt{\kappa} \ll \zeta \ll 1 / \sqrt{\log \kappa}$. This choice of $\zeta$ leads to a magnetic field $O\left(1 / \zeta^{2}\right)$ which motivates the following expansion:

$$
\mathbf{H}_{o}=\frac{\mathbf{H}_{o}^{(0)}}{\zeta^{2}}+\mathbf{H}_{o}^{(1)} \cdots .
$$

Note, however, that although $\mathbf{H}_{o}$ is singular as each vortex line is approached, the singularity occurs in the second term of the expansion. In fact $\mathbf{H}_{o} \sim \log \rho \mathbf{t}$ for small radial distances $\rho$ from a vortex line. The leading term is non-singular and satisfies the following 'averaged' equation:

$$
\nabla^{2} \mathbf{H}_{o}^{(0)}-\mathbf{H}_{o}^{(0)}=-\boldsymbol{\omega},
$$

where $\omega$ is the averaged vortex density (an $O(1)$ term), and will henceforth will be termed the vorticity (details of the averaging process are given in [10]). Assuming that the vortices are all locally parallel we can, by noting that $\mathbf{H}_{o}^{(0)} / \zeta^{2}$ is also the leading order term in the expansion of the non-singular part of the magnetic field $\mathbf{H}_{0, j}$, write down an approximate velocity $\boldsymbol{v}$ for the vorticity $\omega$

$$
\boldsymbol{v}=\frac{2}{\zeta^{2}}\left(\nabla \wedge \mathbf{H}_{o}^{(0)}\right) \wedge \frac{\omega}{|\boldsymbol{\omega}|} .
$$

This, however, assumes that we can neglect the 'comparatively small' curvature term, and while this is true for certain geometries (see, for example, the existence and regularity proofs in [26] and [16]), it is not, as we shall demonstrate, true for all.

The model is then closed by requiring conservation of vorticity which, on rescaling time $t$ with $\zeta^{2} / 2$ and dropping sub- and superscripts, may be written in its entirety as follows:

$$
\left.\begin{array}{c}
\nabla \wedge(\nabla \wedge \mathbf{H})+\mathbf{H}=\boldsymbol{\omega} \\
\nabla \cdot \mathbf{H}=0 \\
\boldsymbol{v}=(\nabla \wedge \mathbf{H}) \wedge \frac{\boldsymbol{\omega}}{|\boldsymbol{\omega}|} \\
\boldsymbol{\omega}_{t}+\nabla \wedge(\boldsymbol{\omega} \wedge \boldsymbol{v})=\mathbf{0}
\end{array}\right\} \text { in } \Omega,
$$

where $\Omega$ is the domain occupied by the superconductor. Outside the superconductor, Maxwell's equations are satisfied:

$$
\left.\begin{array}{l}
\nabla \wedge \mathbf{H}=\mathbf{0} \\
\nabla \cdot \mathbf{H}=0
\end{array}\right\} \text { in } \bar{\Omega}^{c},
$$

and these couple to the interior via standard jump and boundary conditions. When we 
take $\mu$, the magnetic susceptibility, to be constant throughout the whole region, these may be written as follows:

$$
\left.\begin{array}{c}
{[\mathbf{H}]=\mathbf{0}} \\
\nabla \wedge \mathbf{H} \cdot \hat{\boldsymbol{n}}=0
\end{array}\right\} \quad \text { on } \partial \Omega,
$$

where $\hat{\boldsymbol{n}}$ is the outward normal to the boundary. Initial conditions must be given for $\boldsymbol{\omega}$, together with boundary conditions for $\mathbf{H}$ at infinity and for $\boldsymbol{\omega}$ on $\partial \Omega$. If the boundary condition on the vorticity is $\left.\omega \cdot \hat{\boldsymbol{n}}\right|_{\partial \Omega}=0$, it is enough to specify the flux of vorticity in through those sections of the boundary for which $\left.\boldsymbol{v} \cdot \hat{\boldsymbol{n}}\right|_{\partial \Omega}<0$. In line with the work in [9], which demonstrates that an energy barrier exists to the formation of vortices on the boundary, it is reasonable to take this flux to be zero, and we shall do so. The conditions on $\mathbf{H}$ at infinity depend upon the geometry of the domain and should be determined by the field applied to the sample and the total transport current flowing along the body. We shall consider a superconducting slab occupying the region $-b<x<b$; requiring the field to satisfy

$$
\mathbf{H} \rightarrow \mathbf{H}_{-} \quad \text { as } \quad x \rightarrow-\infty \quad \text { and } \quad \mathbf{H} \rightarrow \mathbf{H}_{+} \quad \text { as } \quad x \rightarrow \infty
$$

determines the applied field $\mathbf{H}_{a p p l}=\left(\mathbf{H}_{-}+\mathbf{H}_{+}\right) / 2$ and the average transport current density $\int_{-b}^{b} \mathbf{j}_{\text {trans }} d x /(2 b)$.

\section{Stability of a force-free steady state}

\subsection{The unperturbed solution}

We now look for a one-dimensional steady-state solution to the mean-field model describing a transport current flow, in the presence of vortices, inside the slab $-b<x<b$. For vortices to be present in equilibrium, we must also allow for an external magnetic field. Outside the slab, in the two regions $x<-b$ and $x>b$, Maxwell's equations (1.9) are satisfied by constant magnetic fields $\mathbf{H}=\mathbf{H}_{-}$and $\mathbf{H}=\mathbf{H}_{+}$. In the slab, a general steady state solution may be written down as follows:

$$
\mathbf{H}_{\text {stdy }}=\left(0, H_{2}(x), H_{3}(x)\right) \quad \omega_{\text {stdy }}=(0,0, \omega(x)),
$$

where

$$
\begin{aligned}
& H_{2}(x)=A \sinh (x-d) \quad \text { in } \quad-b<x<b \text {, } \\
& \left.\begin{array}{l}
H_{3}(x)=B \cosh \left(x-a_{1}\right) \\
\omega(x)=0
\end{array}\right\} \text { in } \quad-b<x<a_{1}, \\
& \left.\begin{array}{l}
H_{3}(x)=B \\
\omega(x)=B
\end{array}\right\} \quad \text { in } a_{1}<x<a_{2}, \\
& \left.\begin{array}{l}
H_{3}(x)=B \cosh \left(x-a_{2}\right) \\
\omega(x)=0
\end{array}\right\} \quad \text { in } \quad a_{2}<x<b,
\end{aligned}
$$

Note the presence of the free-boundaries at $x=a_{1}$ and $x=a_{2}$ separating the region of non-zero vorticity from regions of zero vorticity on either side of it. Referring to the jump 
conditions (1.10), it is clear that the exterior fields giving such an interior solution are

$$
\begin{aligned}
& \mathbf{H}_{\text {stdy }}=\mathbf{H}_{-}=\left(0,-A \sinh (b+d), B \cosh \left(b+a_{1}\right)\right) \quad x<-b, \\
& \mathbf{H}_{s t d y}=\mathbf{H}_{+}=\left(0, A \sinh (b-d), B \cosh \left(b-a_{2}\right)\right) \quad x>b .
\end{aligned}
$$

These in turn may be related to the applied magnetic field $\mathbf{H}_{\text {appl }}$ by

$$
\begin{aligned}
\mathbf{H}_{\text {appl }} & =\frac{\mathbf{H}_{-}+\mathbf{H}_{+}}{2} \\
& =\left(0, A(\sinh (b-d)-\sinh (b+d)) / 2, B\left(\cosh \left(b+a_{1}\right)+\cosh \left(b-a_{2}\right)\right) / 2\right),
\end{aligned}
$$

and the transport current density $\mathbf{j}_{\text {trans }}$ averaged over the width of the slab by

$$
\frac{1}{2 b} \int_{-b}^{b} \mathbf{j}_{\text {trans }} d x=\left(0, B\left(\cosh \left(b+a_{1}\right)-\cosh \left(b-a_{2}\right)\right), A(\sinh (b-d)+\sinh (b+d))\right) .
$$

This still leaves some indeterminacy in the choice of unknown parameters $\left(A, B, a_{1}, a_{2}, d\right)$ given any particular values for the applied field and transport current. To completely determine the unknowns, we must also specify the total vorticity trapped inside the slab

$$
\left.\int_{-b}^{b} \omega d x=(0,0, W)=\left(0,0, B\left(a_{2}-a_{1}\right)\right)\right)
$$

Remark Without loss of generality, we can choose $B \equiv 1$. This corresponds to assigning a particular value to the typical vortex spearation $\zeta$.

\subsection{Linear stability of the basic solution}

We now investigate the stability of this class of solution by imposing a small perturbation onto the original solution, so that

$$
\begin{aligned}
& \omega=\omega_{\text {stdy }}+\epsilon(\alpha(x, t) \cos (m z), \beta(x, t) \sin (m z), \gamma(x, t) \sin (m z))+\cdots, \\
& \mathbf{H}=\mathbf{H}_{\text {stdy }}+\epsilon(\phi(x, t) \cos (m z), \theta(x, t) \sin (m z), \psi(x, t) \sin (m z))+\cdots,
\end{aligned}
$$

where we take $\alpha=\beta=\gamma=0$ for $x<a_{1}$ and $x>a_{2}$ and replace the stationary free boundaries at $x=a_{1}$ and $x=a_{2}$ by the moving boundaries

$$
x=a_{1}+\epsilon \chi_{1}(z, t)+\cdots \quad \text { and } \quad x=a_{2}+\epsilon \chi_{2}(z, t)+\cdots,
$$

respectively. We can obtain a linear problem for the evolution of $\alpha, \beta, \gamma, \phi, \theta, \psi$ and $\zeta$ by substituting (2.2) and (2.3) into (1.8)-(1.10), and taking the order $\epsilon$ term. We can then either solve this problem, with given initial data, by use of a Laplace transform, or look for solutions to it with uniform growth rate such that

$$
\alpha(x, t)=e^{\sigma t} \tilde{\alpha}(x),
$$

with $\tilde{\beta}(x), \tilde{\gamma}(x), \tilde{\chi}_{1}(z), \cdots$ similarly defined. For the moment, we opt for the latter choice, which results in the following coupled ODEs for $\tilde{\phi}, \tilde{\theta}$ in $a_{1}<x<a_{2}$ :

$$
\begin{aligned}
& \left(-\tilde{\phi}^{\prime \prime}+\left(m^{2}+1\right) \tilde{\phi}\right)\left(\sigma(\sigma+1)-A^{2} m^{2} \cosh ^{2}(x-d)\right) \\
& \quad=\tilde{\alpha}\left(\sigma(\sigma+1)-A^{2} m^{2} \cosh ^{2}(x-d)\right)=\left(\sigma \tilde{\phi}+A m^{3} \cosh (x-d) \tilde{\theta}\right),
\end{aligned}
$$




$$
\begin{aligned}
& \left(-\tilde{\theta}^{\prime \prime}+\left(m^{2}+1\right) \tilde{\theta}\right)\left(\sigma(\sigma+1)-A^{2} m^{2} \cosh ^{2}(x-d)\right) \\
& \quad=\tilde{\beta}\left(\sigma(\sigma+1)-A^{2} m^{2} \cosh ^{2}(x-d)\right)=-\left(A m \cosh (x-d) \tilde{\phi}+(\sigma+1) m^{2} \tilde{\theta}\right)
\end{aligned}
$$

and

$$
\left.\begin{array}{l}
-\tilde{\phi}^{\prime \prime}+\left(m^{2}+1\right) \tilde{\phi}=0 \\
-\tilde{\theta}^{\prime \prime}+\left(m^{2}+1\right) \tilde{\theta}=0
\end{array}\right\} \quad \text { in }-b<x<a_{1} \text { and } a_{2}<x<b
$$

where

$$
\tilde{\phi}^{\prime}+m \tilde{\psi}=0 \quad \text { in }-b<x<b, \quad \tilde{\alpha}^{\prime}+m \tilde{\gamma}=0 \quad \text { in } a_{1}<x<a_{2} .
$$

In $\bar{\Omega}^{c}$, substitution of (2.2) and (2.3) into (1.9) gives

$$
\left.\begin{array}{l}
-\tilde{\phi}^{\prime \prime}+m^{2} \tilde{\phi}=0 \\
\tilde{\theta}=0 \\
\tilde{\phi}^{\prime}+m \tilde{\psi}=0
\end{array}\right\} \quad \text { in } x<-b \text { and } x>b \text {, }
$$

Requiring continuity of the field $\mathbf{H}$ and the current $\nabla \wedge \mathbf{H}$ across the two moving boundaries $x=a_{1}+\epsilon \exp (\sigma t) \tilde{\chi}_{1}(z)+\cdots$ and $x=a_{2}+\epsilon \exp (\sigma t) \tilde{\chi}_{2}(z)+\cdots$ and linearising onto $x=a_{1}$ and $x=a_{2}$, respectively, amounts to requiring

$$
[\tilde{\phi}]=[\tilde{\theta}]=\left[\tilde{\phi}^{\prime}\right]=\left[\tilde{\theta}^{\prime}\right]=0 \text { on } x=a_{1} \text { and } x=a_{2} \text {. }
$$

On the boundaries between the superconductor $\Omega$ and the exterior $\bar{\Omega}^{c},(1.10)$ is satisfied by requiring

$$
[\tilde{\phi}]=\left[\tilde{\phi}^{\prime}\right]=0, \quad \tilde{\theta}=0, \quad \text { on } x= \pm b .
$$

Finally, we note that the requirement that perturbations remain everywhere small enforces the far-field conditions

$$
\tilde{\phi} \rightarrow 0, \quad \tilde{\theta} \rightarrow 0, \quad \text { as } x \rightarrow \pm \infty
$$

thus closing the eigenvalue problem given by (2.4)-(2.11).

Turning now to the evaluation of the unknown functions $\tilde{\chi}_{1}(z)$ and $\tilde{\chi}_{2}(z)$, which determine the position of the two moving boundaries, we note that the normal velocity of the moving boundary $v_{n}$ is equal to that component of the velocity of the vorticity in the direction of the normal $\boldsymbol{n}$ to the moving boundary. The vorticity velocity $\boldsymbol{v}$ is given by the following expression:

$$
\boldsymbol{v}=\left(\left(-m \tilde{\phi}+\tilde{\psi}^{\prime}+A \cosh (x-d) \tilde{\beta}\right) \sin (m z),(A \cosh (x-d) \tilde{\alpha}+m \tilde{\theta}) \cos (m z), 0\right) .
$$

Using this expression in conjunction with (2.4)-(2.5), and noting that if $\left.\nabla \cdot \omega\right|_{t=0}=0$ is to hold in a weak sense on the boundary then $\left.\boldsymbol{\omega} \cdot \boldsymbol{n}\right|_{t=0}=0$ on the moving boundary, one finds

$$
\tilde{\chi}_{1}(z)=\frac{\tilde{\alpha}\left(a_{1}\right) \sin (m z)}{m}, \quad \tilde{\chi}_{2}(z)=\frac{\tilde{\alpha}\left(a_{2}\right) \sin (m z)}{m} .
$$

The position of the moving boundaries are thus determined by the solution to the eigenvalue problem, and are such that for all subsequent times $\boldsymbol{\omega} \cdot \boldsymbol{n}=0$ (on the moving boundary).

A considerable simplification to the eigenvalue problem (2.4)-(2.11) can be made by 
solving explicitly in the regions $x<a_{1}$ and $x>a_{2}$, and then using these solutions, which contain some arbitrary constants, to derive boundary conditions on (2.4)-(2.5). Doing so in $x<a_{1}$ we find

$$
\tilde{\phi}=P \exp (m(x+b)), \quad \tilde{\theta}=0, \quad \text { in } x<-b,
$$

and, for $-b<x<a_{1}$

$$
\begin{aligned}
\tilde{\phi} & =P \cosh \left(\left(m^{2}+1\right)^{1 / 2}(x+b)\right)+\frac{P m}{\left(m^{2}+1\right)^{1 / 2}} \sinh \left(\left(m^{2}+1\right)^{1 / 2}(x+b)\right), \\
\tilde{\theta} & =S \sinh \left(\left(m^{2}+1\right)^{1 / 2}(x+b)\right),
\end{aligned}
$$

These solutions may then be used to obtain the following boundary condition on $x=a_{1}$ for equations (2.4)-(2.5):

$$
\begin{gathered}
\tilde{\phi}\left(m \cosh \left(\left(m^{2}+1\right)^{1 / 2}\left(b+a_{1}\right)\right)+\left(m^{2}+1\right)^{1 / 2} \sinh \left(\left(m^{2}+1\right)^{1 / 2}\left(b+a_{1}\right)\right)\right) \\
=\tilde{\phi}^{\prime}\left(\cosh \left(\left(m^{2}+1\right)^{1 / 2}\left(b+a_{1}\right)\right)+\frac{m}{\left(m^{2}+1\right)^{1 / 2}} \sinh \left(\left(m^{2}+1\right)^{1 / 2}\left(b+a_{1}\right)\right)\right), \\
\tilde{\theta}\left(m^{2}+1\right)^{1 / 2} \cosh \left(\left(m^{2}+1\right)^{1 / 2}\left(b+a_{1}\right)\right)=\tilde{\theta}^{\prime} \sinh \left(\left(m^{2}+1\right)^{1 / 2}\left(b+a_{1}\right)\right) .
\end{gathered}
$$

By a similar method, one can obtain the other required boundary condition on $x=a_{2}$

$$
\begin{aligned}
& \tilde{\phi}\left(m \cosh \left(\left(m^{2}+1\right)^{1 / 2}\left(b-a_{2}\right)\right)+\left(m^{2}+1\right)^{1 / 2} \sinh \left(\left(m^{2}+1\right)^{1 / 2}\left(b-a_{2}\right)\right)\right) \\
& =-\tilde{\phi}^{\prime}\left(\cosh \left(\left(m^{2}+1\right)^{1 / 2}\left(b-a_{2}\right)\right)+\frac{m}{\left(m^{2}+1\right)^{1 / 2}} \sinh \left(\left(m^{2}+1\right)^{1 / 2}\left(b-a_{2}\right)\right)\right), \\
& \tilde{\theta}\left(m^{2}+1\right)^{1 / 2} \cosh \left(\left(m^{2}+1\right)^{1 / 2}\left(b-a_{2}\right)\right)=-\tilde{\theta}^{\prime} \sinh \left(\left(m^{2}+1\right)^{1 / 2}\left(b-a_{2}\right)\right) .
\end{aligned}
$$

In the next section, we examine the properties of the eigenvalue problem comprising equations (2.4)-(2.5) and boundary conditions (2.12)-(2.13).

\section{Properties of the eigenvalue system}

For simplicity, we now consider only the eigenvalue system arising from those steady solutions to the mean-field model for which the applied field $\mathbf{H}_{a p p l}$ is parallel to the transport current $\mathbf{j}_{\text {trans }}$. This requirement neccesitates that

$$
d=0 \quad \text { and } \quad a_{1}=-a_{2}=-a .
$$

Hence, such steady-state solutions are symmetric about the origin. It is then readily verified that boundary conditions (2.12)-(2.13), when written in the form

$$
\begin{array}{rlrr}
\tilde{\phi}^{\prime}=r_{1} \tilde{\phi} & \text { and } & \tilde{\theta}^{\prime}=\Upsilon_{2} \tilde{\theta} & \text { on } x=-a, \\
\tilde{\phi}^{\prime}=-r_{1} \tilde{\phi} & \text { and } & \tilde{\theta}^{\prime}=-\Upsilon_{2} \tilde{\theta} & \text { on } x=a,
\end{array}
$$

have the property that both $\Upsilon_{1}$ and $\Upsilon_{2}$ are positive.

\section{Non-singular eigenfunctions}

We can now use the above property to find limits on the range, in the $m-\sigma$ plane, in which it is necessary to search for non-singular eigenvalues. Multiplying (2.4) by $\tilde{\phi}$, then adding 


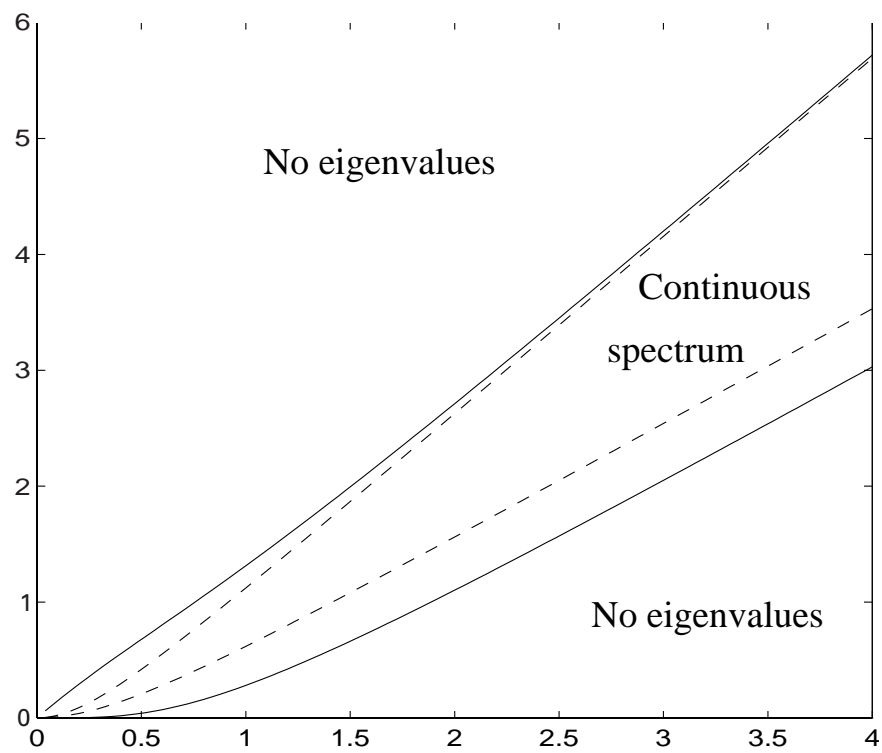

FIGURE 1. Location of eigenvalues in the $m-\sigma$ plane for the case $A=1$ and $a=1$. The four curves plotted on the picture are, from the bottom upwards, $\sigma=\sigma_{3}(m), \sigma=\sigma_{1}(m), \sigma=\sigma_{2}(m)$ and $\sigma=\sigma_{4}(m)$.

the result to (2.5) multiplied by $m^{2} \tilde{\theta}$ and integrating from $x=-a$ to $x=a$ yields the following relation:

$$
\begin{aligned}
\int_{-a}^{a} \tilde{\phi}^{\prime 2}+ & m^{2} \tilde{\theta}^{\prime 2} d x-\left[\tilde{\phi} \tilde{\phi}^{\prime}+m^{2} \tilde{\theta} \tilde{\theta}^{\prime}\right]_{-a}^{a} \\
= & -\left\{\int_{-a}^{a} \tilde{\phi}^{2}\left(m^{2}+1-\frac{\sigma}{\left(\sigma(\sigma+1)-A^{2} m^{2} \cosh ^{2} x\right)}\right)\right. \\
& \left.+m^{2} \tilde{\theta}^{2}\left(m^{2}+1+\frac{(\sigma+1) m^{2}}{\left(\sigma(\sigma+1)-A^{2} m^{2} \cosh ^{2} x\right)}\right) d x\right\} .
\end{aligned}
$$

Reference to (3.2) and (3.3) shows that the left-hand side of (3.4) is always positive. The right-hand side can only be positive if one of the two inequalities

$$
\begin{array}{r}
\sigma /\left(\sigma(\sigma+1)-A^{2} m^{2} \cosh ^{2} x\right)>m^{2}+1, \\
m^{2}(\sigma+1) /\left(A^{2} m^{2} \cosh ^{2} x-\sigma(\sigma+1)\right)>m^{2}+1,
\end{array}
$$

is satisfied in some part of the range $-a<x<a$. Restricting our attention to positive values of $\sigma$, we see that neither of these inequalities is satisfied, and thus that no eigenvalues exist, in those parts of the $m-\sigma$ plane in which either $0<\sigma<\sigma_{3}(m)$ or $\sigma>\sigma_{4}(m)$ (see Figure 1), where

$$
\begin{gathered}
\sigma_{3}(m)=\frac{-\left(1+m^{2} /\left(m^{2}+1\right)\right)+\left(\left(1+m^{2} /\left(m^{2}+1\right)\right)^{2}+4\left(A^{2} m^{2}-m^{2} /\left(m^{2}+1\right)\right)\right)^{1 / 2}}{2}, \\
\sigma_{4}(m)=\frac{-\left(1-1 /\left(m^{2}+1\right)\right)+\left(\left(1-1 /\left(m^{2}+1\right)\right)^{2}+4 A^{2} m^{2} \cosh ^{2} a\right)^{1 / 2}}{2} .
\end{gathered}
$$




\section{Singular Eigenfunctions}

Where $\sigma$ lies in the range $\sigma_{1}(m)<\sigma<\sigma_{2}(m)$,

$$
\sigma_{1}(m)=\frac{-1+\left(1+4 A^{2} m^{2}\right)^{1 / 2}}{2}, \quad \sigma_{2}(m)=\frac{-1+\left(1+4 A^{2} m^{2} \cosh ^{2} a\right)^{1 / 2}}{2},
$$

the coefficient of the highest derivatives in (2.4) and (2.5), $\left(A^{2} m^{2} \cosh ^{2} x-\sigma(\sigma+1)\right)$, has zeros in the range $-a<x<a$ occurring at the positions

$$
x=X_{\sigma}=\operatorname{arcosh}\left(\frac{(\sigma(\sigma+1))^{1 / 2}}{A m}\right), \text { and } x=-X_{\sigma},
$$

(see Figure 1). Eigenfunctions $(\tilde{\phi}, \tilde{\theta})$ with eigenvalues in the range $\sigma_{1}<\sigma<\sigma_{2}$ will therefore have singularities at $x= \pm X_{\sigma}$ and of the form $\left(x-X_{\sigma}\right) \log \left|x-X_{\sigma}\right|$. If the spectrum of the eigenvalues $\sigma$ in this range is discrete, any linear combination of the corresponding eigenfunctions will be singular. Such eigenvalues/eigenfunctions clearly have no bearing on the evolution of the linearised problem describing the growth of small, initially smooth, perturbations to the steady-state solutions of the mean-field model given in $\S 2.1$. However if the spectrum of the eigenvalues is continuous it is possible to form a regular linear combination of the corresponding eigenfunctions of the form

$$
\phi(x, t)=\int_{\sigma_{1}}^{\sigma_{2}} e^{\sigma t} K(\sigma) \tilde{\phi}(\sigma, x) d \sigma, \quad \theta(x, t)=\int_{\sigma_{1}}^{\sigma_{2}} e^{\sigma t} K(\sigma) \tilde{\theta}(\sigma, x) d \sigma,
$$

where $K(\sigma)$ is a smooth function of $\sigma$.

Continuous eigenspectra resulting from singular eigenvalue problems occur in many applications and our treatment follows the methods described by Case \& Drazin [14, 7]. Before proceeding with this treatment, we simplify matters still further by restricting our search to pairs $(\phi, \theta)$ that are even in $x$ such that

$$
\frac{\partial \phi}{\partial x}=0 \quad \text { and } \quad \frac{\partial \theta}{\partial x}=0 \quad \text { on } \quad x=0 .
$$

It is easy, but slightly tedious, to generalise our arguments to $(\phi, \theta)$ which do not satisfy this condition, and since this generalisation leaves our conclusions unchanged, we omit it from the following.

To investigate the possibility of a continuous eigenspectrum it is helpful to introduce the Laplace transforms of $\phi$ and $\theta$, which are defined as follows:

$$
\bar{\phi}(x, p)=\int_{0}^{\infty} e^{-p t} \phi(x, t) d t, \quad \bar{\theta}(x, p)=\int_{0}^{\infty} e^{-p t} \theta(x, t) d t .
$$

We then take the Laplace-transformed version of the linear problem obtained by substituting (2.2) and (2.3) into (1.8)-(1.10) and taking the order $\epsilon$ term. By following steps similar to those outlined in $\S 2.2$, it is possible to show that this Laplace transformed problem may be written in following form:

$$
\begin{aligned}
& \mathscr{L}_{1}(\eta, \varpi)=R(x, p)=\frac{\alpha(x, 0)+\beta(x, 0) \sqrt{(p+1) / p}}{(\sqrt{p(p+1)}+A m \cosh x)}, \\
& \mathscr{L}_{2}(\eta, \varpi)=Q(x, p)=\frac{\alpha(x, 0)-\beta(x, 0) \sqrt{(p+1) / p}}{(\sqrt{p(p+1)}-A m \cosh x)} .
\end{aligned}
$$


Here the linear operators $\mathscr{L}_{1}$ and $\mathscr{L}_{2}$ are defined as follows:

$$
\begin{aligned}
\mathscr{L}_{1}(\eta, \varpi)= & -\frac{d^{2} \eta}{d x^{2}}+\left(m^{2}+1\right) \eta \\
& -\frac{1}{2} \frac{\left(\eta\left(\sqrt{p /(p+1)}-m^{2} \sqrt{(p+1) / p}\right)+\varpi\left(m^{2} \sqrt{(p+1) / p}+\sqrt{p /(p+1)}\right)\right)}{(\sqrt{p(p+1)}+A m \cosh x)}, \\
\mathscr{L}_{2}(\eta, \varpi)= & -\frac{d^{2} \varpi}{d x^{2}}+\left(m^{2}+1\right) \varpi \\
& -\frac{1}{2} \frac{\left(\varpi\left(\sqrt{p /(p+1)}-m^{2} \sqrt{(p+1) / p}\right)+\eta\left(m^{2} \sqrt{(p+1) / p}+\sqrt{p /(p+1)}\right)\right)}{(\sqrt{p(p+1)}-A m \cosh x)},
\end{aligned}
$$

the functions $\varpi$ and $\eta$ are defined in terms of $\bar{\phi}$ and $\bar{\theta}$ by

$$
\eta(x, p)=\bar{\theta}+\sqrt{\frac{p+1}{p}} \bar{\phi}, \quad \varpi(x, p)=\sqrt{\frac{p+1}{p}} \bar{\phi}-\bar{\theta},
$$

and $\varpi$ and $\eta$ satisfy certain homogeneous boundary conditions on $x=0$ and $x=a$. We can write the solution to (3.6) and (3.7) in terms of convolutions involving Green's functions:

$$
\begin{aligned}
\eta(x, p) & =\int_{0}^{a} R(\xi, p) g_{1}(x, p ; \xi)+Q(\xi, p) g_{2}(x, p ; \xi) d \xi \\
\varpi(x, p) & =\int_{0}^{a} R(\xi, p) h_{1}(x, p ; \xi)+Q(\xi, p) h_{2}(x, p ; \xi) d \xi
\end{aligned}
$$

where the Green's function pairs $\left(g_{1}(x, p ; \xi), h_{1}(x, p ; \xi)\right)$ and $\left(g_{2}(x, p ; \xi), h_{2}(x, p ; \xi)\right)$ are obtained by solving

$$
\mathscr{L}_{1}\left(g_{1}(x, p ; \xi), h_{1}(x, p ; \xi)\right)=\delta(x-\xi), \quad \mathscr{L}_{2}\left(g_{1}(x, p ; \xi), h_{1}(x, p ; \xi)\right)=0,
$$

and

$$
\mathscr{L}_{1}\left(g_{2}(x, p ; \xi), h_{2}(x, p ; \xi)\right)=0, \quad \mathscr{L}_{2}\left(g_{2}(x, p ; \xi), h_{2}(x, p ; \xi)\right)=\delta(x-\xi),
$$

respectively. Finally, we can calculate $\bar{\phi}(x, p)$ and $\bar{\theta}(x, p)$, in terms of these Green's functions, and invert to find $\phi(x, t)$ and $\theta(x, t)$ :

$$
\begin{aligned}
\phi(x, t)=\frac{1}{2 \pi i} \int_{0}^{a} \int_{\gamma-i \infty}^{\gamma+i \infty} \frac{e^{p t}}{2}\left(\frac{p}{p+1}\right)^{1 / 2}\left[R ( \xi , p ) \left(g_{1}(x, p ; \xi)\right.\right. \\
\left.\left.+h_{1}(x, p ; \xi)\right)+Q(\xi, p)\left(g_{2}(x, p ; \xi)+h_{2}(x, p ; \xi)\right)\right] d p d \xi, \\
\theta(x, t)=\frac{1}{2 \pi i} \int_{0}^{a} \int_{\gamma-i \infty}^{\gamma+i \infty} \frac{e^{p t}}{2}\left[R ( \xi , p ) \left(g_{1}(x, p ; \xi)\right.\right. \\
\left.\left.\quad-h_{1}(x, p ; \xi)\right)+Q(\xi, p)\left(g_{2}(x, p ; \xi)-h_{2}(x, p ; \xi)\right)\right] d p d \xi .
\end{aligned}
$$

Since $Q(\xi, p)$ has poles in $p$ for $\xi=X_{p}=\operatorname{arcosh}\left((p(p+1))^{1 / 2} / A m\right)$, and the range of $\xi$ is $[0, a]$, the solutions for $\phi$ and $\theta$ will in general exhibit growth rates $e^{\sigma t}$ for 
$\sigma_{1}(m)<\sigma<\sigma_{2}(m)$ (see equation (3.4) for the definitions of $\sigma_{1}(m)$ and $\sigma_{2}(m)$ ). The Green's functions $h_{1}(x, p ; \xi)$ and $h_{2}(x, p ; \xi)$ also exhibit $\left(x-X_{p}\right) \log \left|x-X_{p}\right|$ type singularities because of the singular terms in the left-hand side of (3.7), but these are smoothed out by the integrals in $p$ in equations (3.8) and (3.9). Thus $\phi(x, t)$ and $\theta(x, t)$ are regular in $x$.

\subsection{Summary}

We have shown there is a continuous spectrum of positive eigenvalues $\sigma \in\left(\sigma_{1}(m), \sigma_{2}(m)\right)$ associated with singular eigenfunctions. However, since the spectrum of eigenvalues is continuous, small smooth perturbations added to the steady state solution to the meanfield model (2.1), and of the form given in (2.2)-(2.3), potentially have growth rates $\sigma$ as large as $\sigma_{2}(m) .{ }^{1}$ The problem given by the mean-field model with initial conditions defined by (2.1) and (3.1) is thus ill-posed, a consequence of $\sigma_{2}(m)$ having no upper bound

$$
\sigma_{2}(m) \sim A m \cosh (a) \text { for } m \gg 1 .
$$

\section{A possible regularisation of the mean-field model}

In the previous section we demonstrated that not only are the force free steady states given by (2.1) unstable, but when used for initial data to the mean-field model (1.8), result in an ill-posed problem. Comparison with the behaviour of a single vortex in an applied current reveals why this should be so. It has been shown [23] that, where the current $\mathbf{j}$ has some component in the direction of the tangent to the vortex $\mathbf{t}$, the vortex velocity law

$$
v=\mathbf{j} \wedge \mathbf{t}
$$

leads to an ill-posed problem. If, however, the small self-induced curvature term is retained (see equation (1.5)), such that the vortex now obeys the velocity law

$$
\boldsymbol{v}=\mathbf{j} \wedge \mathbf{t}+(\log \kappa) C \boldsymbol{n},
$$

then the resulting problem, even where $\mathbf{j} \cdot \mathbf{t} \neq 0$, is well-posed. This suggests that in the case of the mean-field model whenever $\mathbf{j} \cdot \boldsymbol{\omega} \neq 0$ it is wrong to neglect the curvature term. We can incorporate this by replacing the velocity $\boldsymbol{v}$ in (1.8) by

$$
\boldsymbol{v}=(\nabla \wedge \mathbf{H}) \wedge \frac{\omega}{|\boldsymbol{\omega}|}+v\left(\left(\frac{\omega}{|\boldsymbol{\omega}|}\right) \cdot \nabla\right)\left(\frac{\omega}{|\boldsymbol{\omega}|}\right),
$$

where the coefficient $v$ is given by

$$
v=\frac{\zeta^{2}}{2} \log \kappa
$$

We now proceed to examine the consequences of adding such a term for the stability of the steady state solution we found in $\S 2.1$, namely (2.1). First, we note that this solution is still a steady state solution of the model with the self-induced curvature term retained in the velocity. Then proceeding exactly as we did in $\S 2.2$, we look for a perturbation to

1 We could find no numerical evidence of a discrete spectrum in the range $\sigma_{2}(m)<\sigma<\sigma_{4}(m)$ suggesting that $\sigma_{2}(m)$ is indeed the maximum growth rate that can occur. 
this solution of the form

$$
\begin{aligned}
& \omega=\omega_{s t d y}+\epsilon \exp (\Sigma t)(\tilde{\alpha}(x) \cos (m z), \tilde{\beta}(x) \sin (m z), \tilde{\gamma}(x) \sin (m z))+\cdots, \\
& \mathbf{H}=\mathbf{H}_{\text {stdy }}+\epsilon \exp (\Sigma t)(\tilde{\phi}(x) \cos (m z), \tilde{\theta}(x) \sin (m z), \tilde{\psi}(x) \sin (m z))+\cdots,
\end{aligned}
$$

and find after pursuing essentially the same steps that $\tilde{\phi}$ and $\tilde{\theta}$ satisfy the same eigenvalue problem as they did there, namely that given by equations (2.4), (2.5), (2.12) and (2.13). The one notable difference is that the growth rate of the perturbation $\Sigma$ is no longer given by the eigenvalue $\sigma$, but related to it as follows:

$$
\Sigma=\sigma-v m^{2} .
$$

This is an important difference and means that whereas the growth rate of the unmodified model grows linearly with $m$ the growth rate for the amended model has a maximum value; for $v \ll 1$ this is given by

$$
\Sigma_{\max } \approx \frac{A^{2} \cosh ^{2} a}{4 v} \text { at } m \approx \frac{A \cosh a}{2 v} .
$$

Thus, it transpires that, at least for this initial data, the modified model is linearly wellposed. However, the steady-state solution is clearly not stable (since $\Sigma_{\max }$ is positive). It proves rather interesting to compare the maximum growth rate for this solution with that for a single vortex in a constant applied current $\mathbf{j}$ (see [23]) for which it is found that

$$
\sigma_{\max } \approx \frac{(\mathbf{j} \cdot \mathbf{t})^{2}}{4 \log \kappa} \quad \text { at } \quad m \approx \frac{(\mathbf{j} \cdot \mathbf{t})}{2 \log \kappa} .
$$

By noting that time has been rescaled with $\zeta^{2} / 2$ in the mean-field model and that $\mathbf{j}_{o}=\nabla \wedge \mathbf{H}_{o} \approx \nabla \wedge \mathbf{H}_{\text {stdy }} /\left(\zeta^{2}\right)$ one can see that the maximum growth rate for the modified mean-field model, and the wavenumber at which this occurs, are the same as they would be for vortices positioned along the lines of maximum longitudinal current $x= \pm a$.

\subsection{Applicability of the model}

A basic assumption of the mean-field approach is that on the scale of vortex separation $\zeta$ vortices are almost parallel. Variations of the vorticity $\omega$ over a lengthscale smaller, or of the same order as $\zeta$, therefore violate this assumption. Equating the wavenumber $m$ at which the maximal growth of the instability occurs (see equation (4.3)) with the length in which longitudinal variations in $\omega$ typically occur, we can see that for validity of the model we require

$$
\frac{2 \pi \zeta \log \kappa}{A \cosh a} \gg O(1)
$$

or in terms of the original units in which $H_{c 2}=\kappa^{2}$ and the penetration depth $\lambda=1$,

$$
j_{\text {long }} \ll \frac{2 \pi \log \kappa}{\zeta},
$$

where $j_{\text {long }}$ gives a measure of the size of the current running parallel to the vorticity (in this case $\left.\zeta^{2} A \cosh a\right)$. 


\section{Remark}

Referring back to the original expansion of the magnetic field (1.7) we see that the expansion for the current is as follows:

$$
\mathbf{j}_{o}=\frac{\mathbf{j}_{o}^{(0)}}{\zeta^{2}}+\frac{\mathbf{j}_{o}^{(1)}}{\zeta}+\cdots .
$$

Note that whereas the second term in the expansion for $\mathbf{H}_{o}$ is $O(1)$, for the current $\mathbf{j}_{o}$ it is of order $(1 / \zeta)$. This is a consequence of the singularity at this order. If we require that variations in $\omega$ occur over a longer lengthscale than vortex separation $\zeta$ we impose such a severe restriction on the longitudinal part of the current that we must be resigned to its being hardly greater than the second term in the expansion for the current $\mathbf{j}_{o}^{(1)} / \zeta$. This term is composed of intervortex currents which give rise to local interaction forces between neighbouring vortices but which average to zero over many vortex separations (see [24]). Where locally dominant, it results in the formation of a vortex lattice with a resistance to shearing motions. However for sufficiently high temperatures, thermal energy is enough to 'melt' a vortex lattice (see, for example, [17]), and in these regimes one might reasonable expect the mean-field approach to hold.

\section{Conclusion}

We started by conjecturing that the mean-field model given in [10] is ill-posed unless the current and vorticity is everywhere perpendicular so that $\mathbf{j} \cdot \boldsymbol{\omega} \equiv 0$. We proceeded to illustrate this conjecture by finding a force-free steady-state solution to the model (for which $\mathbf{j} \wedge \boldsymbol{\omega}=\mathbf{0}$ and $\mathbf{j} \cdot \boldsymbol{\omega} \neq \mathbf{0}$ ) and then conducting a linear stability analysis about this solution. The result of the stability analysis revealed that, with initial data close to the steady-state solution, the mean-field model is indeed ill-posed. In $\S 4$ a regularised model was proposed and was used to repeat the stability analysis of the force-free steady-state solution. This gave an indication of the well-posedness of the new model and showed this force-free steady-state to be unstable. This last result has some inherent interest because there have been various attempts to use force-free steady-states to overcome dissipation in Type-II superconductors. Finally, we discussed the parameter regime over which one would expect the regularised model to hold and noted that, although this might be fairly restrictive for very low temperatures where there is a strong tendency for vortices to form a lattice, for temperatures high enough to 'melt' the vortex lattice the model has a considerably larger domain of validity.

A natural extension to this work would be to investigate the case where a small transport current is applied to a collection of vortices held together in a stiff lattice by larger intervortex currents. Work by Clem [13] on the stability of a single vortex in a circular superconducting cylinder shows that a combination of line tension (the curvature term in the velocity law) and an azimuthal screening current (resulting from a longitudinal applied magnetic field) is enough to stabilise the vortex for sufficiently small longitudinal currents. By analogy with this, we might well expect to find a critical longitudinal current below which the stiff vortex lattice is stable.

The other extreme case, in which a large longitudinal current flows resulting in typical radii of curvature of the vortices with sizes much smaller than the vortex separation, has 
already been investigated in [23] revealing the unfeasability of stable force-free steady states (without pinning) in this regime.

\section{Acknowledgements}

The authors would like to thank Drs S. J. Chapman and J. R. Ockendon for helpful discussions. GR would like to acknowledge the support of an ESF research fellowship and an OCIAM research assistantship.

\section{References}

[1] Abrikosov, A. A. (1957) On the Magnetic Properties of a Superconductor of the Second Kind Sov. Physics JETP, 5, 174.

[2] Campbell, A. M. \& EvetTs, J. E. (1972) Flux vortices and transport currents in Type II superconductors. Adv. in Physics, 21, 199.

[3] Brandt, E. H. (1995) The flux-line-lattice in superconductors. Rep. Prog. Phys., 58, 1465

[4] Brandt, E. H. (1990) Flux diffusion in high- $T_{c}$ superconductors. Zeit. Phys. B., 80, 165.

[5] Brandt, E. H. (1981) Longitudinal critical current in Type II superconductors. I. Helical vortex instability in the bulk. J. Low Temp. Phys. 44(1/2), 33.

[6] Brandt, E. H. (1981) Longitudinal critical current in Type II superconductors. II Helical vortex instability near the surface. J. Low Temp. Phys. 44(1/2), 59.

[7] CASE, K. M. (1960) Stability of inviscid plane Couette flow. Phys. of Fluids, 3(2), 143.

[8] Chapman, S. J., Howison, S. D. \& Ockenden, J. R. (1992) Macroscopic models for superconductivity. SIAM Rev., 34(4), 529.

[9] Chapman, S. J. (1995) Superheating fields of Type II superconductors. SIAM J. Appl. Math. $\mathbf{5 5}(5), 1233$.

[10] Chapman, S. J. (1995) A mean-field model of superconducting vortices in three dimensions. SIAM J. Appl. Math. 55(5), 1259-1274.

[11] Chapman, S. J. \& Richardson, G. (1995) Motion of vortices in type-II superconductors. SIAM J. Appl. Math. 55(5), 1275-1296.

[12] Chapman, S. J., Rubinstein, J. \& Schatzman, M. (1996) A mean-field model of superconducting vortices. Euro. J. Appl. Math. 7, 97-118.

[13] Clem, J. R. (1977) Spiral vortex interaction instability in Type-II superconductors. Phys Rev. Lett. 38, 1425.

[14] Drazin, P. G. \& ReID, W. H. (1981) Hydrodynamic Stability. Cambridge University Press.

[15] Du, Q., Gunzburger, M. D. \& Peterson, J. S. (1992) Analysis and approximation of the Ginzburg-Landau model of superconductivity. SIAM Rev. 34, 54.

[16] Elliott, C. M., Shätzle, R. \& Stoth, B. (1998) Viscosity solutions of a degenerate parabolicelliptic system arising from the mean-field theory of superconductivity. Arch. Rat. Mech. 145, 99.

[17] Fisher, D. S. (1980) Flux line melting in thin-film superconductors. Phys. Rev. B, 22, 3.

[18] Ginzburg, V. L. \& Landau, L. D. (1950) On the theory of superconductivity. J.E.T.P. 20, 1064.

[19] Gor'Kov, L. P. \& Eliashburg, G. M. Generalisation of the Ginzburg-Landau equations for non-stationary problems in the case of alloys with paramagnetic impurities. Sov. Phys. J.E.T.P. 27, 328.

[20] Gor'kov, L. P. \& Kopnin, N. B. (1971) Viscous vortex flow in superconductors with paramagnetic impurities. Sov. Phys. J.E.T.P. 33, 1251.

[21] Hu, C. R. \& Thompson, R. S. (1972) Dynamic structures of vortices in superconductors. II. $H \ll H_{c 2}$. Phys Rev. B, 6(1), 110. 
[22] Peres, L. \& Rubinstein, J. (1993) Vortex dynamics in U(1) Ginzburg-Landau models. Physica $D, 64(1-3), 299-309$.

[23] Richardson, G. (1997) Instability of a superconducting line vortex. Physica D, 108, 139.

[24] Richardson, G. (1995) Vortex Motion in Type-II Superconductors. DPhil Thesis, University of Oxford.

[25] Schmid, A. (1966) A time dependent Ginzburg-Landau equation and its application to the problem of resistivity in the mixed state. Physik der Kondensierten Materie, 5, 302.

[26] Schätzle, R. \& Styles, V. M. (1999) Analysis of a Mean Field Model of Superconducting Vortices. Euro. J. Appl. Math. to appear. 